\title{
Multiple Anti-submarine Helicopters' Cooperative Front Submarine Detecting Research
}

\author{
Li Xinshu' ${ }^{\mathrm{a}}$, Li Weibo ${ }^{\mathrm{b}}$, Mao Zhongyang ${ }^{\mathrm{c}}$, Zhang Yang ${ }^{\mathrm{d}}$ \\ Naval Aeronautical Engineering Institute Yantai, Shandong, China 264001 \\ axinssh@sina.com, ${ }^{\mathrm{b}}$ sunlight2637@163.com, ${ }^{\mathrm{c}}$ kinleb227@126.com, ${ }^{\mathrm{d}} \mathrm{ZY}$ 83051@sina.com
}

\begin{abstract}
Keywords: Front submarine detecting; Warship formation; Anti-submarine position configuration; Flight process; Maneuver avoidance; Torpedo attack
\end{abstract}

\begin{abstract}
This paper is aiming at solving the problem of front submarine detecting combat of multiple ship-borne anti-submarine helicopters in the process of naval warship formation. By analyzing the launch range of submarine-launched torpedo, torpedo attack launch position models are established under two conditions of ideal condition and formation evasive maneuver, and the position configuration of front submarine detecting area is obtained. On this basis, multiple helicopters front submarine detecting methods are analyzed in the process of formation flight, and front submarine detecting force demand and probing probability model are established. Finally, the influence of the submarine-launched torpedo launch position, anti-submarine force size, the number of detection and the size of front submarine detecting area on the probability of discovery is calculated. The results show that it is appropriate when front submarine detecting area is 20 nautical miles in the front of the formation, $20 * 30$ nautical miles size, and three anti-submarine helicopters.
\end{abstract}

\section{Introduction}

Surface warships are vulnerable to enemy submarine attacks during voyage, especially when enemy submarine uses position ambush tactics. Modern advanced submarine has nice mute performance [1-2]. Generally hover or micro-speed navigation and other tactical measures are used after it gets into ambush position, making passive sonar difficult to find its trail; and surface ships' active sonar detection distance is limited, and it is difficult to implement effective confrontation. Therefore, carrier-based anti-submarine helicopter goes for the implementation of escort anti-submarine mission with escort. It is one of the effective means to fight against such threats and the formation of flight safety.

There has been lots of domestic and foreign research on carrier-based anti-submarine helicopter anti-submarine warfare [3-12]. More research is on the search methods used by anti-submarine helicopter [3-7] or the search equipment [8-12], and the research work on carrier-based anti-submarine helicopter implementation of front submarine detecting operations, especially anti-submarine position configuration, is still less. At present, only qualitative analysis is carried out in the area of escort area, accompanied by escort method, and lack of necessary model support and simulation verification. Based on the background of warship formation, this paper studies the multiple carrier-based submarine detecting helicopters front submarine detecting combat on the basis of modeling and analyzing front submarine detecting area configuration.

\section{Carrier-based anti-submarine helicopter front submarine detecting}

The front-end method is one of the main methods of carrier-based anti-submarine helicopter front submarine detecting, and it is an anti-submarine tactic against enemy submarine position ambush tactic. Position ambush means that the submarine may set ambush positions in warships on the possible route. When gets into its foothold, warship formation carries out formation reconnaissance or implementation of torpedo and missile attack waiting for the right opportunity. The tactics are almost ideal for all performances of submarines, especially for submarines with less maneuverability. However, the use of this tactic needs to be more accurately informed of the 
formation's route information.

Front submarine detecting generally sets anti-submarine helicopter configuration in a certain area of anti-submarine route in front of formation. The aim is to always provide anti-submarine alert and sufficient defense for the formation in voyage, discover the enemy submarine and attack, or force the enemy submarine to get away from the ambush position. The primary problem in front submarine detecting is anti-submarine position setting and anti-submarine force configuration, which are the main research contents of this article.

Equipment for anti-submarine helicopter to search underwater target are: dipping sonar, sonar buoy, and magnetometer and so on. Dipping sonar can be used repeatedly, active or passive conversion is easy, and detection distance is far, and through anti-submarine helicopter maneuver, detection can be done in any position in anti-submarine area. Compared with other equipment, it is more suitable for front submarine detecting. Therefore, this article mainly studies the related issues of multiple helicopters dipping sonar carrying out front submarine detecting.

\section{Analysis on the modeling of carrier-based anti-submarine helicopter front submarine detecting area}

In submarine threat area, in order to fully guarantee the safety of naval fleet, anti-submarine force should search the sea area in front of the formation in a certain distance and to eliminate submarine threat as far as possible. General front submarine detecting method is: anti-submarine force in a linear configuration is in the vertical route direction, which constitutes a linear anti-submarine interception zone, and anti-submarine zone's average forward movement speed is consistent with that of the formation, so as to ensure the relative stability of anti-submarine position. This method is called front submarine detecting line. It has the following problems: enemy submarine can be circumvented, thus escaping detection, and then threaten the formation security; it is difficult to make multiple search in the same area; the effective search area is small, so it is difficult to provide sufficient anti-submarine depth; multiple helicopters anti-submarine organization and command are more complex.

In order to solve these shortcomings, front submarine detecting line is changed into front submarine detecting search area, namely: in formation front route, determine a number of adjacent front submarine detecting areas, one of which is shown in Figure 1. Anti-submarine force makes repeated search in advance of a certain time in the region. When the distance between the formation and anti-submarine zone is $D_{\text {s0 }}$, anti-submarine force completes the search on front submarine detecting area. This method changes dynamic front submarine detecting search line into a relatively static area check anti-submarine; and anti-submarine force can make searches within the region repeatedly, so that the enemy submarine is difficult to effectively avoid search, and it is easy to achieve the operation attempts such as drive or attack submarine, and ensure formation voyage safety.

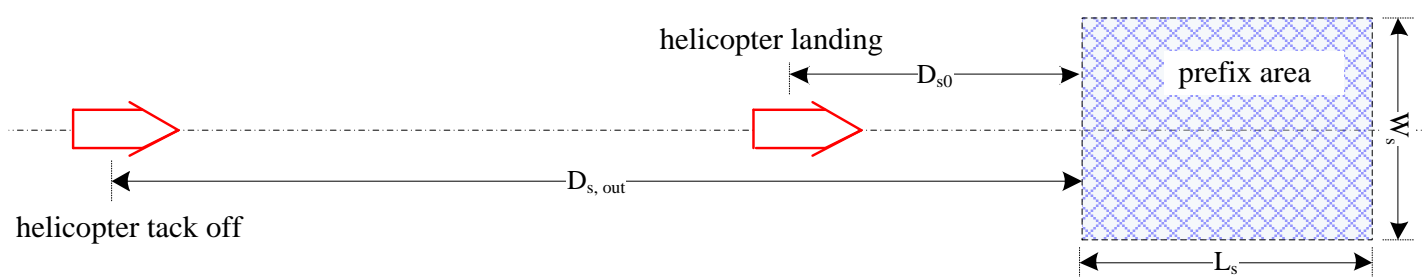

Figure 1 Front submarine detecting area position

The key issue of front submarine detecting area is to determine the length $L_{s}$ and width $W_{s}$ of the anti-submarine zone, and end the search at what distance $D_{s 0}$ to ensure the safety of formation and improve the effectiveness of anti-submarine force. As formation voyage is of a higher speed, and generally there are surface ships with stronger anti-submarine capability in formation, so the width of front anti-submarine area should not be too large. But in order to defend submarine flank attack, anti-submarine area should cover winged torpedo firing position. 
To protect its own security and reduce the probability of being found, enemy submarine usually attacks in the maximum range of torpedo. Enemy submarines use position ambush tactics, and the main weapon of attack ship warship formation is submarine-launched torpedo. The following mainly starts modeling analysis from enemy submarine's possible torpedo attack position.

A. Ideal position modeling of enemy submarine torpedo attack

When enemy submarine launches torpedo, torpedo should meet the target within the voyage, otherwise it cannot hit; therefore, for targets in different locations with different speed, the maximum distance of the torpedo is different. Therefore, there is a need to get the maximum range of torpedo under different attack angle, resulting in torpedo attack position.

Torpedo attack process is more complex. In order to simplify the analysis, first consider the situation of the torpedo from the maximum launch range under ideal conditions, namely: naval warship formation sails in straight line sailing with uniform speed, and enemy submarine can accurately know the ship's target position, and after the launch of torpedo move toward the meeting point. Therefore, if the naval fleet is sailing at an average speed $V_{S}$; enemy submarine torpedo voyage $R_{\text {tor }}$, the distance between the launch point and the target $D_{0}$, at point $\mathrm{B}$, the average speed $V_{\text {tor }}$, movement towards the meeting point, meet with the ship target at point $\mathrm{A}$. The space positional relationship at horizontal level is shown in Figure 2.

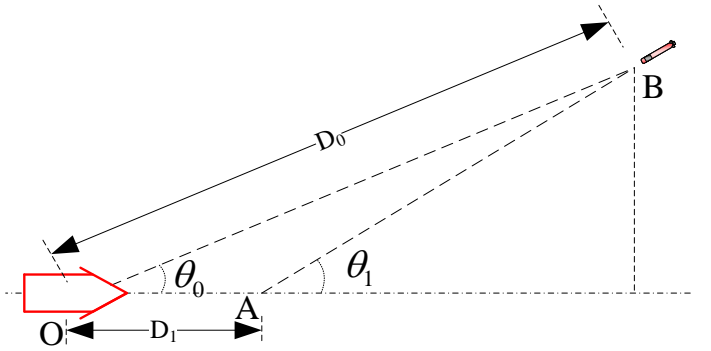

Figure 2 Torpedo attack position diagram

By geometric relations, get

$\left\{\begin{array}{l}V_{\text {tor }} t \cos \theta_{1}=D_{0} \cos \theta_{0}-D_{1} \\ V_{\text {tor }} t \sin \theta_{1}=D_{0} \sin \theta_{0} \\ D_{1}=V_{S} t\end{array}\right.$

And limited by torpedo voyage, get

$V_{\text {tor }} t<\kappa \cdot R_{\text {tor }}$

In the formula, $\kappa$ is torpedo effective launch range coefficient, which is a positive number of not more than 1 . Take $\kappa$ as 0.8 , that is, launch torpedo at $80 \%$ of the torpedo voyage. At this time, the launch distance is called the effective launch distance.

According to (1) and (2), torpedo launch position can be resolved, that is, under the maximum launch distance $D_{0, \text { max }}$ with different $\theta_{0}$. It is possible to hit the target only if the torpedo is launched within that distance.

B. Torpedo attack position under the influence of warship formation maneuvering

In actual combat, if torpedo attacks are found, warship formation usually takes turn maneuvering, etc. to avoid torpedoes. Obviously, if enemy submarine attacks in torpedo's maximum attack range, then the ship fleet takes some avoidance measures, which will significantly reduce the probability of torpedo hit. Therefore, in order to improve hit probability, enemy submarine makes less attack in torpedo's maximum range. The following is the modeling for torpedo attack position when warships take steering avoidance.

Formation avoidance and torpedo attack process is complex, so it is difficult to model the entire process. By simplification, the following assumptions are made:

(1) Formation can find the torpedo within scope $d_{f}$, and once find torpedo, immediately take avoidance maneuver; 
(2) Formation only takes one avoidance maneuver; avoidance direction is to get the largest distance from torpedo movement;

(3) The torpedo can always find its target. Taking into account the enemy submarine sonar detection process, assume formation turn time $t_{\text {delay }}$, the torpedo can get formation information, and begin to chase its goal. Submarine torpedo is mainly wire guide, so the submarine can pass the target information detected to the torpedo, but submarine detection of target takes some time, so make this assumption.

On the basis of the hypothesis, if ship warship formation find a torpedo at some time away the distance $d_{f}$, direction $\psi_{A B}$, as shown in Figure 3, the formation immediately turn $\varphi_{S}$ with radius of $r_{S}$, the course turns from $\psi_{S}$ to $\psi_{E G}$, and then travel in straight line; after formation turn $t_{\text {delay }}$, torpedo arrives at point $\mathrm{B}$, and begins to turn $\varphi_{T}$ with radius $r_{T}$, the course turns from $\psi_{T}$ to $\psi_{H G}$, and finally meets the target at point $\mathrm{G}$. So there is

$$
\begin{aligned}
& \frac{d_{1}+r_{S} \varphi_{S}}{V_{S}}-t_{\text {delay }}=\frac{d_{2}+r_{T} \varphi_{T}}{V_{T}} \\
& d_{T B}+d_{2}+r_{T} \varphi_{T}<\kappa \cdot R_{\text {tor }}
\end{aligned}
$$

In it, $d_{T B}$ is the displacement of the torpedo movement to point $\mathrm{B}$ after launching, according to the value and $\psi_{T}$, torpedo firing point coordinates can be calculated, resulting in the distance of torpedo movement; $d_{1}, d_{2}, \varphi_{T}$ are unknown. According to the triangular relationship, get the solution.

Depending on the range values of $d_{T B}$, as well as the azimuth $\psi_{T}$, the launch point of the torpedo can be reversed.

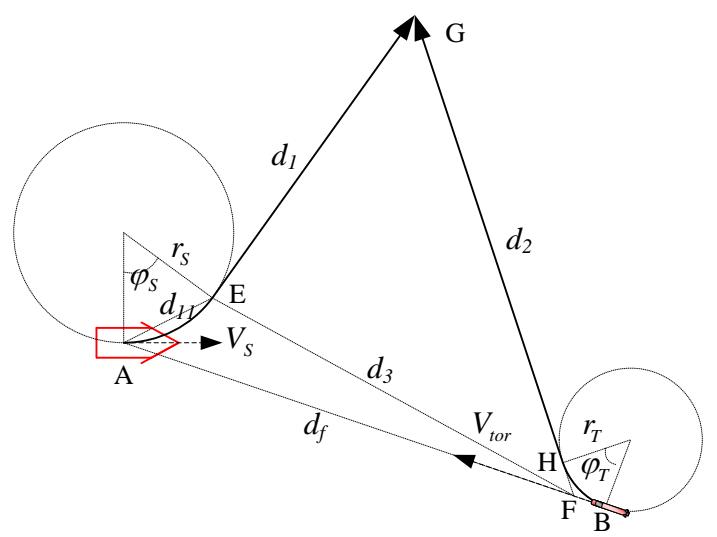

Figure 3 Torpedo attack diagram when the formation is making steering avoidance

In triangular EFG, there are

$$
\left\{\begin{array}{l}
d_{1} \cos (\angle G E F)+d_{2}^{\prime} \cos (\angle G F E)=d_{3} \\
d_{1} \sin (\angle G E F)=d_{2}^{\prime} \sin (\angle G F E) \\
d_{2}^{\prime}=d_{2}+r_{T} \tan \left(\varphi_{T} / 2\right)
\end{array}\right.
$$

In the formula, $\angle G E F$ can be obtained from $\psi_{F E}$ and $\psi_{E G}, \angle G F E$ can be obtained from $\psi_{F E}$ and $\psi_{H G}$.

In triangular AEF, according to cosine theorem, there is

$$
d_{3}=\sqrt{d_{11}^{2}+d_{f 1}^{2}-2 d_{11} d_{f 1} \cos (\angle E A F)}
$$

In it, $d_{11}=\varphi_{S} r_{S}, d_{f 1}=d_{f}-r_{T} \tan \left(\varphi_{T} / 2\right), \angle E A F$ can be obtained from $\psi_{A B}$ and $\varphi_{S}$; In the same way, according to cosine theorem, $\angle E F A_{\text {can }}$ be got, but then according to azimuth angle $\psi_{A B}$, the azimuth angle $\psi_{F E}$ can be got. 


\section{Determination of front submarine detecting area}

Based on the previous analysis of the modeling, we can see that the position of front submarine detecting area should take into account factors such as formation voyage data, enemy submarine performance and its torpedo attack distance. In addition, when ship-based anti-submarine helicopters perform anti-submarine in anti-submarine area, whether it will affect escort anti-submarine ship sonar detection or be affected should also be considered. The size of front submarine detecting area is mainly limited by the number of ship-borne anti-submarine helicopters, the performance of anti-submarine helicopters, the performance of snare equipment, and marine environment and other factors.

In order to make reliably attack and safely go out of the ambush position, the probability of enemy submarine attack in the effective launch of torpedo is high. Therefore, front submarine detecting area should cover these areas, and as far as possible find the enemy submarine before the torpedo is launched, and attack or drive it. Therefore, anti-submarine area should cover the ideal position of torpedo attack corresponding to $\kappa$ is 1 and 0.8 and torpedo attack position under the influence of the formation maneuver.

\section{Conclusion}

Modern silent submarines, as well as the use of position ambush tactics, constitute a serious underwater threat against fleet warships in voyage. Therefore, in order to effectively protect formation flight safety, implement front submarine detecting through the use of carrier-based anti-submarine helicopter can make up for the deficiency of anti-submarine capability in formation navigation. In this paper, the following conclusions are obtained through modeling and simulation:

(1) Warships formation uses evasive maneuvering strategy which compresses torpedo firing position of the enemy submarine. The simulation parameters show that the maximum circumference of the torpedo is reduced by about 10 nautical miles compared with no evasive maneuvering.

(2) Under the simulation parameters, the ideal attack position of the torpedo is $20 \sim 40$ nautical miles in front of the warship formation. Therefore, the front submarine detecting area should be set at 20 to 40 nautical miles in front of the formation; if the front submarine detecting area is $20 * 30$ nautical miles, the number of anti-submarine helicopters is appropriately three;

(3) The increase in the number of anti-submarine helicopter detection can improve the probability of discovery, but when the number of detection exceeds a certain value, the increase is not obvious.

Front submarine detecting area position and the size of the anti-submarine zone, the number of anti-submarine force are the key problems of front submarine detecting of ship-borne anti-submarine helicopter; this paper establishes relevant calculation model to provide decision-making basis for the development of warship formation conductor and implementation of front search.

\section{References}

[1] WangYu, YaoYaoZhong. Word Navy Submarine[M]. Beijing: National Defense Industry Press, 2006:130.

[2] WangWeiSheng. “Cang Long”- New Development of Japanese Submarine Technology [J]. Modern Navy, 2009,4:52-55

[3] Danskin. J., A helicopter versus submarine search game[J]. Operations Research, 1968,16:509-517.

[4] Edmund Cheong Kong Chuan. A Helicopter submarine Search Game[D]. Master's Thesis of Naval Postgraduate School,1988.

[5] E Qun; MA Yuan-liang; LIU De-cai. Ship-Heli Cooperating Area Checking Anti-Submarine Searching Mode[J]. Fire Control and Command Control, 2009,34(10):115-116. 
[6] Zhang Shengyun, Zhang An, Wang Lian-zhu, est. A Study on Searching for Submarine by Antisubmarine Airfly[J]. Fire Control and Command Control, 2006,31(1): 70-74.

[7] Dong Chaofeng. Study on Searching Method of Helicopter on Submarine with Definite Direction and Velocity[J]. Fire Control and Command Control, 2008,22(5):39-41.

[8] Martin J. Multiplying the effectiveness of helicopter ASW sensors[J]. Sea Technology, 2006,47(11): 33-36.

[9] Wu Ming. Simulation research and model building based on checking submarine search of multi-aircraft dipping sonar[J]. Computer Engineering and Design, 2010,31(3):676-679.

[10] Wu Fang, Yang Rijie. Model Building and Simulation Based on Definite Second Time Submarine Search by Multi-aircraft Dipping Sonar[J]. Acta Aeronautica et Astronautica Sinica, 2009,30(10):1948-1953. (in Chinese)

[11] Bai Jing, Han Liang. Search method of dipping sonar on shipborne antisubmarine aircraft[J].Journal of Beijing University of Aeronautics and Astronautics, 2007,33(3):282-285.

[12] Wu Fang, Yang Rijie, Sang Chunlong. Building and Simulating Definite Second Time Submarine Search Models of Dipping Sonar Based on Transcendental Target Moving Course[J].Journal of System Simulation,2009,21(20):6630-6633.

[13] Sun MingTai, est. Air Antisubmarine Tactical[M]. Beijing: Military Science Press. 2003. 90-93, 88.

[14] Zhao XiaoZhe, Shen ZhiHe. Navy Operational mathematic model [M]. Beijing: National Defense Industry Press, 2004, 40-41.

[15] MK-48 Torpedo. http://www.fas.org/man/dod-101/sys/ship/weaps/mk-48.htm. 2011, 0914. 